\title{
Interprofessional Scenario-Based Learning for New Graduates in a Regional Setting: A Pilot Study
}

Priya Martin

Cunningham Centre, Darling Downs Hospital and Health Service, preeyamartin99@gmail.com

Matthew Newby

Central Queensland Hospital and Health Service, newby_88@hotmail.com

Monica Moran

Central Queensland University, monica.moran@cqu.edu.au

Matthew Browne

Central Queensland Uinversity, m.browne@cqu.edu.au

Saravana Kumar

University of South Australia, saravana.kumar@unisa.edu.au

Follow this and additional works at: https://nsuworks.nova.edu/ijahsp

Part of the Medical Education Commons

\section{Recommended Citation}

Martin P, Newby M, Moran M, Browne M, Kumar S. Interprofessional Scenario-Based Learning for New Graduates in a Regional Setting: A Pilot Study. The Internet Journal of Allied Health Sciences and Practice. 2016 Jan 01;14(3), Article 6.

This Manuscript is brought to you for free and open access by the College of Health Care Sciences at NSUWorks. It has been accepted for inclusion in Internet Journal of Allied Health Sciences and Practice by an authorized editor of NSUWorks. For more information, please contact nsuworks@nova.edu. 


\title{
Interprofessional Scenario-Based Learning for New Graduates in a Regional Setting: A Pilot Study
}

\begin{abstract}
Introduction: Globally there is an increased emphasis on the provision of high quality healthcare and improved productivity under mounting financial constraints. Interprofessional collaboration and practice are considered crucial in promoting teamwork and optimising patient outcomes. However, there is a lack of structured, evaluated interprofessional learning opportunities for graduates in regional and rural health services in Australia. This pilot study reports on a novel interprofessional new graduate learning program that was developed and implemented to address the lack of structured post-qualification interprofessional learning opportunities in the Central Queensland Hospital and Health Service. Methods: Twenty-one participants enrolled in the six-month program, which consisted of six ninety-minute sessions. A mixed method exploratory design was used to collect data using the Interprofessional Socialization and Valuing Scale before and after the program, and a reflective summary at program completion. Results: Results indicated that participation in the program had a positive effect on participants' attitudes, beliefs and behaviours regarding interprofessional practice as measured on the Interprofessional Socialization and Valuing Scale. Various themes about the usefulness of the program to participants were elicited from the reflective summary data. These included enhanced understanding of interprofessional practice, increased confidence in own professional role, improved understanding of the roles of other professions, increased collaboration with others, improved patient outcomes and improved networking with colleagues. Conclusions: It was concluded that the new graduate interprofessional learning program was a feasible and effective way to facilitate interprofessional learning among health graduates in a regional health service.
\end{abstract}

\section{Author Bio(s)}

- Priya Martin, BOT, MS (Psychotherapy\&Counseling), works as an advanced clinical educator at the Cunningham Centre, Darling Downs Hospital and Health Service, in Queensland, Australia. Prior to this she was occupational therapy clinical education support officer at Central Queensland Hospital and Health Service. She is currently completing a PhD through the University of South Australia.

- Matthew Newby, BHlthSci (Nutrition\&Dietetics), is an Accredited Practicing Dietitian (APD) in Australia and Registered Dietitian (RD) in the United Kingdom. He worked as a senior dietitian and clinical educator for the Central Queensland Hospital and Health Service before moving to England to work as a Locum Specialist Dietitian for the NHS.

- Monica Moran, DSocSc, MPhil(OccThy), GCClinED, DipCOT, is an associate professor and discipline lead of the occupational therapy program at Central Queensland University in Rockhampton, Queensland. She researches in the areas of interprofessional education, role emerging professional practice and the consumer experience of healthcare.

- Matthew Browne, PhD, received his doctorate in cognitive psychophysiology from Griffith University in 2001. He has worked as a biostatistician in Japan, Germany and Australia, subsequently working as an applied mathematician at CSIRO, Australia's peak research organisation. His focus is on quantitative and methodological tools to solve applied problems in the social sciences 
- Saravana Kumar, B.App.Sc (Physio) MPT(Manipulative\&Sports) PhD, is a Senior Lecturer and Research Leader at the School of Health Sciences, University of South Australia. He is also a registered Physiotherapist in South Australia.

\section{Acknowledgements}

The authors would like to thank Dr Zephanie Tyack, Queensland Health for her expert advice on the research design of the program. Also, the authors thank Ms Kate Moore for her input into the development of the program, and Ms Kate Moore, Ms Courtney Dixon and Ms Joanne Lowry for assisting with the facilitation of sessions. 


\title{
IJAHSP \\ The Internet Joưnal of Allied Health Sciences and Practice
}

Dedicated to allied health professional practice and education

Vol. 14 No. 3 ISSN 1540-580X

\section{Interprofessional Scenario-Based Learning for New Graduates in a Regional Setting: A Pilot Study}

\author{
Priya Martin, BOT, MS ${ }^{1}$ \\ Matthew Newby, BHIthSci² \\ Monica Moran, DSocSc ${ }^{3}$ \\ Matthew Browne, $\mathrm{PhD}^{3}$ \\ Saravana Kumar, B.App.Sc (Physio), MPT (Manipulative and Sports), PhD4 \\ 1. Cunningham Centre, Darling Downs Hospital and Health Service \\ 2. Central Queensland Hospital and Health Service \\ 3. Central Queensland University \\ 4. University of South Australia, International Centre for Allied Health Evidence
}

Australia

\begin{abstract}
Introduction: Globally, there is an increased emphasis on the provision of high quality healthcare and improved productivity under mounting financial constraints. Interprofessional collaboration and practice are considered crucial in promoting teamwork and optimising patient outcomes. However, there is a lack of structured, evaluated interprofessional learning opportunities for graduates in regional and rural health services in Australia. This pilot study reports on a novel interprofessional new graduate learning program that was developed and implemented to address the lack of structured post-qualification interprofessional learning opportunities in the Central Queensland Hospital and Health Service. Methods: Twenty-one participants enrolled in the six-month program, which consisted of six ninety-minute sessions. A mixed method exploratory design was used to collect data using the Interprofessional Socialization and Valuing Scale before and after the program, and a reflective summary at program completion. Results: Results indicated that participation in the program had a positive effect on participants' attitudes, beliefs, and behaviours regarding interprofessional practice as measured on the Interprofessional Socialization and Valuing Scale. Various themes about the usefulness of the program to participants were elicited from the reflective summary data. These included enhanced understanding of interprofessional practice, increased confidence in own professional role, improved understanding of the roles of other professions, increased collaboration with others, improved patient outcomes, and improved networking with colleagues. Conclusions: It was concluded that the new graduate interprofessional learning program was a feasible and effective way to facilitate interprofessional learning among health graduates in a regional health service.
\end{abstract}

\section{INTRODUCTION}

Despite significant investments in health workforce reform over recent years, the tendency of the various health professions to act in isolation from each other or in competition with each other have inhibited efforts to address workforce challenges. ${ }^{1}$ While a multidisciplinary care approach is considered a key element to quality health care delivery, inadequate training in communication and collaboration has demonstrated that representation of various members of the health care team does not necessarily equate to mutual understanding and respect.2-4 This also impedes the ability of healthcare team members to work collaboratively to provide effective and efficient health care delivery. Recent studies demonstrate growing support for Interprofessional Collaborative Practice (IPCP). 3,5,6 This term endorsed by the World Health Organisation (WHO) goes beyond professions merely working alongside each other, emphasising mutual problem solving and collaborative practice in order to achieve better patient care. IPCP is viewed as a desirable end-goal of interprofessional learning and education. ${ }^{7}$ 
Interprofessional learning (IPL) has been described as an ongoing, active learning process between different cultures and health care disciplines embracing lifelong learning and adult learning principles. ${ }^{7}$ Interprofessional education (IPE) occurs when two or more professionals learn with, from, and about each other to improve collaboration and quality of care. ${ }^{7}$ There is a growing body of evidence demonstrating that IPL and IPE can reduce the tendency of professions to work in silos and to increase capacity for collaborative practice. This leads to more effective healthcare delivery, reduced healthcare costs, and ultimately improved patient care. ${ }^{8}$

A significant growth in IPE initiatives in the pre-registration years is evident in the literature, and IPL is becoming embedded in health professionals' curricula. ${ }^{8}$ However, few opportunities exist in the post-qualification period to explore the full scope of other professionals' roles, values, and attitudes. ${ }^{3}$ A lack of IPE initiatives for qualified professionals is of concern, as any investment in IPE in the pre-registration years could be undermined if graduate health professionals are exposed to uni-professional and hierarchical practice in health settings. ${ }^{9}$ Furthermore, it has been argued that IPCP is essential in health settings to sustain the required cultural shift in the health workforce towards interprofessional practice. ${ }^{5}$

Regional and rural health services in Australia face challenges of staff recruitment and retention. ${ }^{10,11}$ Those employed in these areas can be isolated and often have relatively few professional development opportunities compared to their urban counterparts. ${ }^{11-}$ ${ }^{13}$ Central Queensland Hospital and Health Service (CQHHS) is one region that has been facing significant health and workforce challenges. ${ }^{14}$ Serving a population of around 228,000 people, CQHHS has a diverse geographical footprint ranging from regional cities to remote townships..$^{15}$ One way to address some of the geographical and workforce issues is to make innovative and contextualised professional development opportunities available locally. To this end, a systematic New Graduate Interprofessional Learning program (henceforth referred to as the NGIPL program or the program) was developed and implemented in 2013. CQHHS aspires to provide Interprofessional organizational (IPO) interventions that include changes at the organizational level to enhance collaboration and the quality of care. ${ }^{16}$ The effectiveness of such programs in regional and rural areas has only been minimally researched to date. ${ }^{17}$ Therefore, this paper aims to evaluate the effectiveness of the NGIPL program.

\section{THE NGIPL PROGRAM}

A recent report on Australian health reforms calls for development of a culture of interprofessional practice across the healthcare sector, highlighting the need for interprofessional programs in the workplace. ${ }^{18}$ The first two authors conducted a literature review on IPL and IPE to inform the development of the NGIPL program. Australian literature was primarily used in order to contextualise the program to the National Health Service and workforce models. In addition, international literature was consulted to gather further evidence and support for program content and delivery style. Because of the limited number of evaluated graduate interprofessional education initiatives in the workplace, both pre- and post-registration programs were reviewed.

A number of factors were considered in the development of the program. Three clinical education staff from different professional backgrounds (Nutrition and Dietetics, Occupational Therapy, and Social Work) within the district collectively reviewed the literature and developed the NGIPL Program. Principles of adult learning were reviewed to prepare facilitators and assist in program planning. Facilitators agreed on defined goals and expectations for the program and developed structured session plans to accompany each session. An element of flexibility was incorporated in the session plans to cater for participant needs. Consultation with an expert in pre-registration interprofessional education (third author) was undertaken to validate the program content and structure. The University of Toronto's Points for Interprofessional Education System (PIPES) tool was used to assess if the program met criteria to be considered interprofessional learning. ${ }^{19}$ These strategies enabled the program facilitators to develop content that was designed to promote cross-disciplinary practice and contextualised to the CQHHS settings.

Various strategies were employed to ensure that the program was interactive, interesting, and practical. Participants participated in real world, case scenario-based learning activities. They were encouraged to bring their experiences to the discussions. Reflective practice was facilitated during group discussions. Group rules and expectations were identified by participants during the first session and revisited on commencement of each subsequent session to promote a safe learning environment. A scenariobased approach formed the backbone of the structure of the program, as this approach has been shown to be an effective learning and teaching method that is also enjoyable for participants. ${ }^{20}$ Further information about the program has been attached as Appendix A.

The stated learning objectives of the NGIPL program were to:

- Gain better understanding of other health professions

- Improve confidence in own profession

- Improve interprofessional collaboration

- Reduce "tribalism" of professions

(c) The Internet Journal of Allied Health Sciences and Practice, 2016 
- Share knowledge and experiences

- Access professional development

- Acquire post-qualification IPL opportunities

- Network with other health professionals

\section{METHODS}

This study used a mixed method exploratory pre-post design. ${ }^{21}$ Mixed methods research involves use of both quantitative and qualitative research methods. An exploratory pre-post design involves collecting data before and after the intervention (i.e the program). The Interprofessional Socialisation and Valuing Scale (ISVS) was administered prior to the commencement of the program and at program completion. In addition, each participant completed a reflective summary of their learning experience at the completion of the program.

\section{Ethics}

Ethical approval was obtained from the Central Queensland Hospital and Health Service Human Research Ethics Committee (Ref - HREC 13/QCQ/4). Site specific approvals were further obtained from the above-stated institutional review committee.

\section{Participants}

The NGIPL program was open to all allied health new graduates in their first two years of practice in CQHHS. Support for rolling out the program was obtained from the Director of Clinical Support Services in CQHHS. The program was advertised with the support of clinical educators, professional directors, and team leaders. Individual invitations were sent to all the allied health new graduates across the region.

\section{Procedure}

Data were collected through a basic demographic data form at baseline, through the ISVS at baseline and post-baseline, as well as a reflective summary at program completion. Baseline data were collected before session one. Post-baseline data were collected after the last session. Two authors who were not involved in the program design and delivery assisted with the analysis and interpretation of the evaluation data in order to avoid bias.

\section{Outcome Measures}

Demographic data collected included, age, gender, profession, geographical location, practice setting, years of experience in current profession, and prior interprofessional education.

The ISVS provided quantitative data. The ISVS measured changes to attitudes, beliefs, and behaviours of participants regarding interprofessional collaboration. The ISVS was chosen because it explores key areas aligning closely with the aims of the program. The ISVS questionnaire consists of 34 items, 24 of which have been demonstrated to possess a strong factor structure corresponding to three major subscales: self-perceived ability to work with others (self-perception of ability and skills), value in working with others (enhanced appreciation and understanding of interprofessional practice), and comfort in working with others (comfort in interprofessional team interaction). ${ }^{22}$

The semi-structured reflective summary administered at program completion provided qualitative data on participants' learnings and experiences from the program. The summary consisted of five open-ended questions to prompt participants to reflect on the program. These questions were developed by the first two authors based on the literature review findings and the authors' reflections on the program content. Provision was made for additional comments and de-identified data were collected using a unique code only identifiable by the participant. The reflective summary questions have been attached as Appendix B.

\section{RESULTS}

Twenty-one participants enrolled in the program, representing nine health professions including clinical measurements, medical imagining, nutrition and dietetics, occupational therapy, pharmacy, physiotherapy, podiatry, psychology, and speech pathology. The majority of participants were from the central hub and participated in the program face-to-face. Four participants participated via videoconference from outer centres. Participants were from various practice settings including acute hospital, rehabilitation, mental health, sub-acute, and chronic care. Seventy-six per cent of participants attended three or more sessions. The group was mainly female (81\%) and $67 \%$ of participants were in their first year of practice. Prior IPE had been undertaken by $91 \%$ of participants. Of this, $78 \%$ reported that IPE occurred during the pre-registration years. Only $9 \%$ of participants had been involved in IPE in the post-registration years. 


\section{Quantitative Results}

Eleven participants completed the ISVS questionnaire at baseline and post-baseline. Despite the limited sample size, the repeated measures (pre- and post- training evaluation) design provided scope for a quantitative evaluation of the effectiveness of the program. Figure 1 shows group means and standard errors for the three ISVS subscales before and after the intervention. Repeated measures tests of mean differences were accomplished using linear mixed effects modelling, with model comparison using chi-square statistics. There was an overall increase in the mean ISVS subscale response score of $1.01,95 \% \mathrm{Cl}=[0.74$; $1.26]$, in the three self-report measures from pre- to post-training, $x^{2}(1)=42.4, p<.001$. Mean responses per subscale also differed significantly; $x^{2}(2)=20.76, p<.001$, with respondents rating "appreciation of interprofessional practice" lower than the other two subscales both before and after the intervention. Comparison of a main effects model with an interaction (pre/post by ISVS subscale) model was non-significant, $x^{2}(2)=0.36, p=.863$, suggesting that the positive effects of training were similar across each ISVS subscale.

Figure 1: Group means and standard errors for the three ISVS subscales before and after the intervention

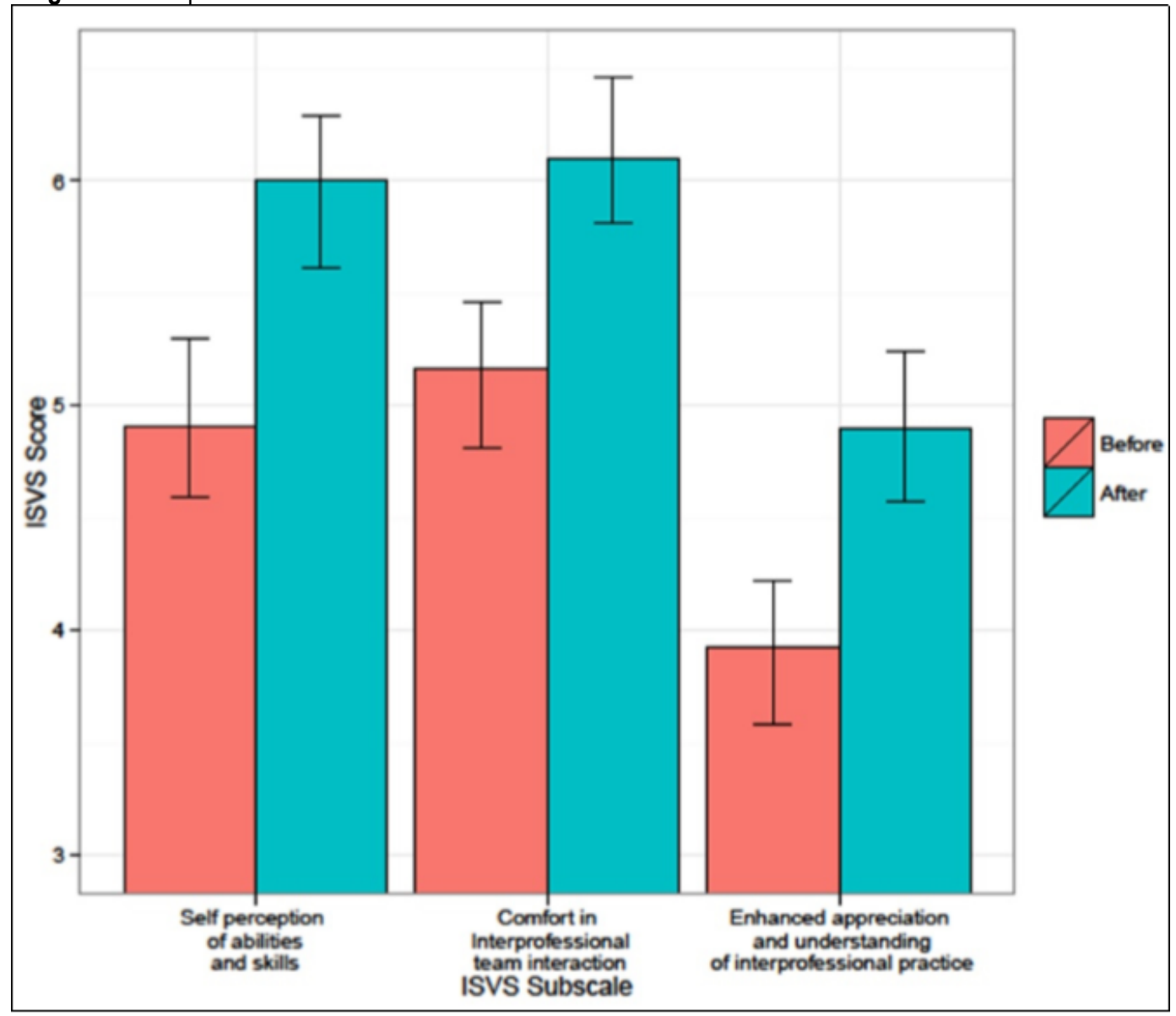

\section{Qualitative Results}

Qualitative data were analysed using inductive content analysis as described by Elo and Kyngas. ${ }^{23}$ There are three phases in inductive content analysis - preparation, organizing, and reporting. In the preparation phase, the researcher becomes familiar with the data by reading it several times. The researcher strives to make sense of the data and to learn what is going on. The organization phase includes open coding (notes and headings are written in the text while reading it), creating categories (by grouping the data) and abstraction (formulating a general description of the research topic from the categories generated). These categories and themes are then reported. ${ }^{23}$ This approach allowed themes to emerge that were grounded in the data rather than imposing pre-defined themes. A simple count was used to elicit the most commonly occurring themes which best represented the participants' views and experiences. The first two authors undertook this process individually and then met to compare their themes to promote intercoder reliability. Furthermore, the third author completed this process independently on a proportion of the data to validate the results. 
Seventeen participants completed the reflective summaries at program completion. Content analysis of the data from the reflective summaries illuminated participant perceptions of the usefulness of the program. A list of themes and sample comments are discussed below:

\section{Enhanced understanding of interprofessional practice}

The majority of participants reported that the NGIPL enhanced their understanding of interprofessional practice, improved their openness to other professions' views, and enhanced their ability to think globally. Participants reported that the NGIPL program provided an opportunity to see things from other professionals' viewpoints. They stated that the program opened their eyes to the process and benefits of interprofessional practice to themselves and to service delivery.

One participant supported this with the following statement:

"The program has introduced me to the term and concept of interprofessional practice. I recognise the benefit in working alongside other health disciplines for the goal of most efficient use of resources, timely processes and improved patient outcomes..."

\section{Increased confidence in own professional role}

Another theme that emerged was that participation in the NGIPL program promoted deeper understanding of one's professional scope and increased confidence in one's own profession. Participants reported that participation in the NGIPL program improved their confidence in explaining the role of their own profession to others and in understanding how their role fits in with others. Furthermore, participants reported that the NGIPL program provided a forum where they explained their role to others in the settings they were employed in, which in turn made them more confident about explaining what they do in their roles.

One participant said:

"The NGIPL program provided more confidence in what my profession contributes."

\section{Improved understanding of the roles of other health professions}

Participants reported that participation in the NGIPL program resulted in greater understanding of the roles of other health professions. This was especially true for the professions that participants didn't work with on a regular basis. Participants expressed that in clinical settings, there are limited formal opportunities to get to know about other professionals' roles. They stated that this gap was addressed by the NGIPL program which provided a dedicated forum and protected time to formally learn about other professionals' roles.

One participant said:

"It (NGIPL program) has enhanced my understanding of the scope and roles of health professionals in different hospital settings (acute vs. rehab) and with professionals I don't work with on a daily basis (e.g., podiatrist)."

Another participant stated:

"I now appreciate the role of some of the less prominent allied health professions on the wards (e.g., medical imaging, podiatry, clinical measurements) and how their input can shape the patient's journey."

\section{Increased collaboration with others}

A theme emerged around the NGIPL program leading to increased confidence in communicating, collaborating, and referring to other health professionals, within and across heath settings.

One participant commented:

"...(NGIPL program) has made me more open to the viewpoints of other disciplines and looking forward for ways to collaborate together and help each other's goals...more aware of any stereotypes that I may have towards other disciplines." 


\section{Improved patient outcomes}

Most participants reported perceptions of participation in the NGIPL program resulting in improved patient outcomes through improved holistic care. They agreed that improved collaboration and teamwork resulted in better outcomes for patients.

One participated said:

"The program has influenced my practice in relation to client care by highlighting the importance of good interprofessional practice, which is vital in providing the best quality care for clients/patients. By having good interprofessional practice clients are receiving care that is holistic and client-centred."

\section{Improved networking}

Morning tea was organised after the first and the last sessions with an aim to promote networking between the participants. Participants reported that NGIPL provided an opportunity to network with other new graduates and strengthen professional relationships.

One participant said:

"I have enjoyed being part of the program, for the learning aspect, but also for the opportunity to network. It is nice now to be able to see familiar faces around the hospital of graduates I otherwise would not have met."

Another participant stated:

"It can be daunting working in a new place as a new graduate. It was nice to see some friendly faces in unfamiliar parts of the hospital."

Other themes that also emerged from the reflective summaries included the value and benefits on the scenario-based learning format, the flexible nature of the program, and the enjoyment and thought-provoking nature of the warm-up, wind-down, and reflective activities.

\section{DISCUSSION}

Results suggest that participation in the NGIPL program led to increased confidence in one's own profession, improved understanding of the roles of other professions, and increased collaboration with team members from other professions. Similar to the findings from Pearson and Pandya's study (which explored views of participants regarding a series of IPL sessions carried out in a primary care setting in the UK), our findings support the premise that IPL can promote a change in attitudes or stereotypes among professionals. ${ }^{5}$ The NGIPL program evaluation appeared to confirm the feasibility and value of a structured IPL program in a non-metropolitan healthcare setting.

The NGIPL program success can be attributed to a number of factors. These include interactive format of sessions, small group discussions and activities, use of adult learning principles, use of a scenario-based format, and having a patient-centred focus. These factors have been found to result in positive experiences reported by the participants in previous program evaluations. ${ }^{5}$ In addition, enthusiasm of the facilitators and participants are likely to have also contributed to the success of this program.

Exposure to other professions through IPE is required to support interprofessional collaboration. It is an essential element to the concept of learning with, not just about, other professions. The goal of the program is not to "inform" about other professions, but rather to develop process-oriented skills for learning with other professions. Smith and Pilling have suggested that a good range of representation of health professions is essential to maximise IPL during the sessions. ${ }^{3}$ The NGIPL program included representatives from many but not all health professions. To address this, the facilitators invited a guest speaker from one of the missing professions to facilitate discussions and answer questions pertaining to that profession. Measures such as this contributed to the positive outcomes of the program.

Establishing a safe learning environment was considered to be crucial while delivering the NGIPL program because participants had varying levels of experience and qualifications, raising the potential issue of power imbalance. The facilitators used a range of strategies to ensure that the participants felt safe during discussions and activities. Ground rules and group expectations were agreed upon at program commencement and were reviewed at the beginning of each session. Pre-existing stereotypes about professional groups can limit as well as act as a barrier to collaboration. This in turn is likely to lead to a lack of respect for other professions. Establishing a safe learning environment contributed to increased collaboration and mutual respect. Interactive

(C) The Internet Journal of Allied Health Sciences and Practice, 2016 
activities during sessions one and two aimed at breaking professional stereotypes and acknowledging the important contribution made by each profession.

Interprofessional learning can promote thinking outside the normal parameters explored in uni-professional education and practice, which can improve one's ability to think critically. ${ }^{24}$ Scenario-based learning further encourages this critical thinking, translating the theory discussed into practice. Billet highlights the importance of shared understandings (intersubjectivity) amongst health care workers and the contribution of joint problem solving and goal directed exercises as mechanisms to enhance these shared understandings in health care teams. ${ }^{25}$ Many participants commented positively on the scenario-based learning format used in the NGIPL sessions that required shared problem solving and increased shared understandings across professions. Small group learning with a patient-centred focus has also been shown to be an effective way of promoting IPL. ${ }^{5}$ The NGIPL program similarly used a scenario-based format, which was found to be an effective way of promoting IPL.

Protected time for facilitators and participants is crucial to the success of such IPL programs in the health setting. ${ }^{6}$ The NGIPL program has highlighted the necessity for protected time for facilitators to engage in program development, planning, delivery, and evaluation and for participants to participate in the program and the evaluation. Based on our experience with the NGIPL program, we would argue that increased organisational support for this form of professional development is a worthwhile investment.

\section{LIMITATIONS}

Seventy-six percent of participants attended three or more sessions. Major contributors to non-attendance of participants were recreational leave and workload commitments. Other studies have noted that participants no longer felt "new" five to six months post-employment, and at that point tend to perceive decreased benefits in programs targeted at graduates. ${ }^{3}$ This may have contributed to decreased attendance, particularly for those in their second year of practice. Smith and Pilling, found that sessions of two-hour duration for four-to-six months post-employment were appropriate for graduate support programs. ${ }^{3}$ Based on the NGIPL program attendance and dropout rate, it is recommended that such programs be run within a shorter timeframe, especially in regional and rural areas where staff turnover issues are prominent.

Ideally, IPL is maximised when healthcare professions are represented from all the health professions. The NGIPL program did not include nursing or medical graduates. Although including all professions is acknowledged as beneficial to the concept of collaboration and promotion, the inclusion of nursing and medical graduates was beyond the scope of the program developers' roles. There exists a need, however, for implementing and evaluating IPL initiatives in the wider healthcare team, especially in non-metropolitan settings.

The retention of facilitators and participants over the duration of the program was found to be challenging. Staff recreational leave and transfers to other roles were found to be common reasons for the turnover. Limiting the NGIPL program to three or four months rather than six months may be helpful in addressing the facilitator turnover and participant dropout issues in regions that consistently face such workforce challenges. Lastly, the impact of the NGIPL program on graduate retention rates in CQHHS was not investigated. Further studies evaluating the impact of IPL programs on graduate retention rates in regional and rural health services are warranted.

\section{CONCLUSIONS}

This study has added to the evidence base that IPL programs in the new graduate context can enhance the attitudes, beliefs, and behaviours of new graduates across a range of health professions regarding interprofessional practice. Behavioural changes reported by participants include transfer of IPL to their practice setting that can be mapped to a level three change on the Kirkpatrick's framework. ${ }^{26}$ This study has also demonstrated that IPL programs are a feasible way of promoting IPE within regional and rural health services. An interactive, scenario-based format has been shown in this study to be an effective method for facilitating the translation of interprofessional knowledge and skills into practice. Sustaining interprofessional organisational (IPO) interventions support from all levels of leadership throughout the organisation is necessary to facilitate change and ensure that programs such as the NGIPL can continue. ${ }^{16}$

\section{References}

1. Frenk J, Chen L, Bhutta ZA, Cohen J, Crisp N, Evans T, et al. Health professionals for a new century: transforming education to strengthen health systems in an interdependent world. The Lancet. 2010;376(9756):1923-58. [PMID: 21112623]

2. Greenstock LN, Brooks PM, Webb GR, Moran MM. Taking stock of interprofessional learning in Australia. The Medical Journal of Australia. 2012;196(11):707. [PMID: 22708770]

3. Smith RA, Pilling S. Allied health graduate program - supporting the transition from student to professional in an interdisciplinary program. Journal of Interprofessional Care. 2007;21(3):265-76.[PMID: 17487705]

(C) The Internet Journal of Allied Health Sciences and Practice, 2016 
4. Fox A, Reeves S. Interprofessional collaborative patient-centred care: a critical exploration of two related discourses. Journal of Interprofessional Care. 2015;29(2):113-8. [PMID: 25180630]

5. Pearson D, Pandya H. Shared learning in primary care: Participants' views of the benefits of this approach. Journal of Interprofessional Care. 2006;20(3):302-13.[PMID: 16777797]

6. Watkin A, Lindquist S, Black J, Watts F. Report on the implementation and evaluation of an interprofessional learning program for inter-agency child protection teams. Child Abuse Review. 2009;18:151-67.

7. Stone J. Interprofessional Collaborative practice (IPCP) - Definitions and terminology, attempting to speak the same language. ACT: ACT Health; 2009.

8. Curriculum Renew for Interprofessional Education in Health. Interprofessional Education: a National Audit. Report to Health Workforce Australia. Sydney, NSW: The Interprofessional Curriculum Renewal Consortium, Australia; 2013.

9. Dunston R. Progress Report - the 'interprofessional' in Australian health professional education: current activity and recommendations for action. Sydney, NSW: Centre for Research in Learning and Change, University of Technology; 2012.

10. Commonwealth Department Rural and Regional Health of Australia. National Strategic Framework for Rural and Remote Health. Final report of the Rural Health Standing Committee. Canberra, ACT: Standing Council on Health; 2012.

11. Hays RB. Interprofessional education in rural practice: how, when and where? Rural and Remote Health 2008;8:939. [PMID: 18452367]

12. Little F, Brown L, Grotowski M, Harris D. Nourishing networks: an interprofessional learning model and its application to the Australian rural health workforce. Rural and Remote Health 2012;12:2022. [PMID: 23121159]

13. Martin $\mathrm{P}$, Abernathy $\mathrm{L}$, Kumar $\mathrm{S}$. Making the most of what you have: Challenges and opportunities from funding restrictions on health practitioners' professional development. The Internet Journal of Allied Health Sciences and Practice [Internet]. 2013;11(4). Available from: http://ijahsp.nova.edu/articles/vol11num4/kumar_11-4.htm.

14. Frakes KA, Brownie S, Davies L, Thomas J, Miller ME, Tyack Z. Experiences from an interprofessional student-assisted chronic disease clinic. Journal of Interprofessional Care. 2014;28(6):573-5. [PMID: 24841001]

15. Central Queensland Hospital and Health Service. 2013-2014 Annual Report. Queensland: CQHHS; 2014.

16. Reeves S, Goldman J, Gilbert J, Tepper J, Silver I, Suter E, Zwarenstein M. A scoping review to improve conceptual clarity of interprofessional interventions. Journal of Interprofessional Care. 2011;25:167-74. [PMID: 21182439]

17. Chau J, Denomme J, Murray J, Cott C. Inter-professional education in the acute-care setting: the clinical instructor's point of view. Physiotherapy Canada. 2009;63(1):65-75. [PMID: 22210981]

18. Brownie S, Thomas J, McAllister L, Groves M. Australian health reforms: enhancing interprofessional practice and competency within the health workforce. Journal of Interprofessional Care. 2014;28(3):252-3. [PMID: 24476107]

19. Points for interprofessional Education System (PIPEs) Allocation [Internet]. Centre for Interprofessional Education: University of Toronto; 2009 March [cited 2015 April 1]. Available from:

http://umanitoba.ca/programs/interprofessional/media/PIPEs_Allocation_Accompanying_Document_March_2009_with_\%2 8C\%29_\%282\%29.pdf.

20. Thistlethwaite JE, Davies D, Ekeocha S, Kidd JM, MacDougall C, Matthews P, et al. The effectiveness of case-based learning in health professional education. A BEME systematic review: BEME Guide No. 23. Medical Teacher. 2012;34(6):421-44. [PMID: 22578051]

21. Creswell JW. Designing Research. In Research Design: Qualitative, Quantitative and Mixed Methods Approaches. California: Sage; 2009.

22. King G, Shaw L, Orchard CA, Miller $S$. The interprofessional socialization and valuing scale: a tool for evaluating the shift toward collaborative care approaches in health care settings. Work. 2010;35(1):77-85. [PMID: 20164627]

23. Elo S, Kyngas H. The qualitative content analysis process. Journal of Advanced Nursing. 2007;62(1):107-15. [PMID: 18352969]

24. Zimmerman SD, Short LGF, Hendrix EM. Impact of interdisciplinary learning on critical thinking using case study method in allied health care graduate students. Journal of Allied Health. 2011;40(1):15-8. [PMID: 21399847]

25. Billet S. Securing intersubjectivity through interprofessional workplace learning experiences. Journal of Interprofessional Care. 2014;28(3):206-11. [PMID: 24571207]

26. Barr H, Koppel I, Reeves S, Hammick M, Freeth D. Effective Interprofessional Education: Argument, Assumption and Evidence. Oxford: Blackwell; 2005. 


\section{Appendix A: The NGIPL Program Outline}

The NGIPL program consisted of six 90-minute sessions delivered monthly between April and September 2013. Sessions were based at the central hub in CQHHS, with videoconferencing made available to link participants from other sites in the health service. Major themes explored throughout the program included interprofessional orientation, eliminating professional stereotypes, teamwork/role definition, and improvements in communication. Although each session promoted a single major theme, sessions were integrated, and content was revisited throughout the program using different modes.

The first two sessions focussed on getting to know each other's professions, understanding the context of interprofessional learning and teamwork. Scenario-based activities were utilised in session three, four and five. Clinical educators from a variety of professions developed the case scenarios, in order to make the content relevant to all professions. The primary focus of the case scenarios was to facilitate teamwork and problem solving, and the identification of potential areas for interprofessional collaboration. A case study was outlined and semi-structured questions and activities were facilitated using a client-centred approach. The group was also asked to identify all opportunities for interprofessional collaboration, in order to highlight the potential for team engagement. An evolving case-study format of a client with Stroke was used to simulate a patient's journey over the three sessions, through acute, sub-acute, and community settings. This approach aimed to offer opportunities for participants to learn more about their own and others' professional roles in various settings. Session six revisited the major themes of the program and aimed to highlight the interprofessional growth made by participants. Morning tea was organised after sessions one and six to promote networking.

Two to three allied health clinical educators, including the first two authors, facilitated each session. Facilitators were from different professional backgrounds (Nutrition and Dietetics, Occupational Therapy, Pharmacy and Social Work) to reinforce the importance of interprofessional collaboration. Most facilitators were also part-time clinicians thus ensuring that content was relevant and relatable. Care was taken to ensure that facilitators were not line managers of any participants, in order to support perceptions of a safe-learning environment. Facilitators gained informal feedback from participants between sessions. They also regularly debriefed and reflected on the sessions together in order to better inform the delivery of subsequent sessions. 


\section{Appendix B: NGIPL Reflective Summary Questions}

1. What impact, if any, has the NGIPL program had on your understanding of interprofessional practice?

2. What impact, if any, has the NGIPL program had on your beliefs, behaviours, and attitudes towards other disciplines within the healthcare setting?

3. Outline ways in which the NGIPL program has influenced your practice in relation to patients/client care.

4. Outline ways in which the NGIPL program has influenced your practice working within the multi-interprofessional team.

5. Provide details about the knowledge, skills and opportunities you have gained from the NGIPL program and how you were able to utilise these in your current role.

6 . Additional comments. 